\title{
Efeitos do uso de polifenóis na terapia do Câncer de Cabeça e Pescoço: Revisão
}

\section{integrativa}

\author{
Effects of polyphenol use in Head and Neck Cancer therapy: Integrative review \\ Efectos del uso de polifenoles en la terapia del Cáncer de Cabeza y Cuello: Revisión integral
}

Recebido: 02/07/2021 | Revisado: 09/07/2021 | Aceito: 12/07/2021 | Publicado: 23/07/2021

Iago Ramirez

ORCID: https://orcid.org/0000-0001-8266-1352 Universidade Federal de Alfenas, Brasil

E-mail: iagoramirez01@gmail.com

Lais Quelen Feitoza

ORCID: https://orcid.org/0000-0002-0776-5933 Universidade Federal de Alfenas, Brasil

E-mail: laisfeitoza2@gmail.com

Lélio Fernando Ferreira Soares

ORCID: https://orcid.org/0000-0003-4174-2943

Universidade Federal de Alfenas, Brasil

E-mail: leliosoaresss@hotmail.com

Fábio de Souza Terra

ORCID: https://orcid.org/0000-0001-8322-3039

Universidade Federal de Alfenas, Brasil

E-mail: fabio.terra@unifal-mg.edu.br

\begin{abstract}
Resumo
Os cânceres de cabeça e pescoço (CCP) apresentam uma grande taxa de morbimortalidade, mesmo após avanços tecnológicos na terapia oncológica convencional. Assim, o estudo de terapias coadjuvantes, como a utilização dos polifenóis, apresenta resultados potenciais no tratamento dos CCP. Essa revisão teve por objetivo identificar os efeitos do uso dos polifenóis no tratamento dos CCP. Foi realizado uma revisão integrativa, a partir da ferramenta PICo, realizando a busca em quatro bases de dados eletrônicas, utilizando os termos "Polifenois", "Câncer de Cabeça e Pescoço" e "Terapia", além de suas correspondências em inglês e espanhol. O total de 25 artigos compuseram a amostra do estudo. A estratégia de extração de dados buscou identificar principalmente o composto, mecanismo de ação, principais desfechos, nível de evidência e delineamento dos estudos. O delineamento de estudo mais prevalente foi "revisão" e "in vitro" e a revisão identificou que os compostos mais utilizados foram Curcumina e Resveratrol. Quase unanimemente, os estudos apontaram para efeitos antineoplásicos dos polifenóis no CCP, ativando mecanismos de morte celular e redução do tumor, por diversas vias. Desse modo, tendo em vista o efeito das terapias convencionais para CCP, bem como seu desfecho clínico, o uso de polifenóis constitui uma opção potencial e promissora instigando o desenvolvimento de pesquisar que abordem detalhadamente os mecanismos celulares, efeitos adversos e dosagens.
\end{abstract}

Palavras-chave: Neoplasias de cabeça e pescoço; Polifenois; Terapêutica.

\begin{abstract}
Head and neck cancers (HNC) have a high morbidity and mortality rate, even after technological advances in conventional cancer therapy. Thus, the study of adjuvant therapies, such as the use of polyphenols, presents potential results in the treatment of HNC. This review aimed to identify the effects of using polyphenols in the treatment of HNC. An integrative review was carried out using the PICo tool, performing the search in four electronic databases, using the terms "Polyphenols", "Head and Neck Cancer" and "Therapy", in addition to their correspondence in English and Spanish. A total of 25 articles comprised the study sample. The data extraction strategy sought to identify mainly the compound, mechanism of action, main outcomes, level of evidence and study design. The most prevalent study design was "review" and "in vitro" and the review identified that the most used compounds were Curcumin and Resveratrol. Almost unanimously, the studies pointed to antineoplastic effects of polyphenols in HNC, activating mechanisms of cell death and tumor reduction, through several ways. Thus, considering the effect of conventional therapies for HNC, as well as its clinical outcome, the use of polyphenols is a potential and promising option, instigating the development of research that addresses in detail the cellular mechanisms, adverse effects and dosages.

Keywords: Head and neck neoplasms; Polyphenols, Therapeutics.
\end{abstract}




\begin{abstract}
Resumen
Los cánceres de cabeza y cuello (CCC) tienen una alta tasa de morbilidad y mortalidad, incluso después de los avances tecnológicos en la terapia convencional del cáncer. Así, el estudio de terapias adyuvantes, como el uso de polifenoles, presenta potenciales resultados en el tratamiento del CCP. Esta revisión tuvo como objetivo identificar los efectos del uso de polifenoles en el tratamiento del CCP. Se realizó una revisión integradora mediante la herramienta PICo, realizando la búsqueda en cuatro bases de datos electrónicas, utilizando los términos "Polifenoles", "Cáncer de cabeza y cuello" y "Terapia", además de su correspondencia en inglés y español. Un total de 25 artículos componen la muestra de estudio. La estrategia de extracción de datos buscó identificar principalmente el compuesto, el mecanismo de acción, los principales resultados, el nivel de evidencia y el diseño del estudio. El diseño de estudio más prevalente fue "revisión" e "in vitro" y la revisión identificó que los compuestos más utilizados fueron la curcumina y el resveratrol. Casi unánimemente, los estudios apuntaron a los efectos antineoplásicos de los polifenoles en el PCC, activando los mecanismos de muerte celular y reducción de tumores, de varias formas. Así, considerando el efecto de las terapias convencionales para la PCC, así como su resultado clínico, el uso de polifenoles es una opción potencial y prometedora, que impulsa el desarrollo de investigaciones que aborden en detalle los mecanismos celulares, los efectos adversos y las dosis.
\end{abstract}

Palabras clave: Neoplasias de cabeza y cuello; Polifenoles; Terapéutica.

\title{
1. Introdução
}

Os cânceres de cabeça e pescoço (CCP) representam um grupo variado de neoplasias malignas que acometem região oral, faríngea e laríngea e é a sétima malignidade mais comum no mundo (Alterio et al., 2019). Aproximadamente 90\% desses tumores são carcinomas espinocelulares (CEC). Os fatores de risco mais conhecidos para seu desenvolvimento são a associação do tabaco e do álcool, infecção por papilomavírus humano (HPV), excesso de gordura corporal e outros associados à ocupação, como exposição ao sol (Cohen et al., 2018). Geralmente, estas neoplasias apresentam um prognóstico ruim pois, na maioria das vezes, o diagnóstico é feito quando os tumores estão em estágios avançados, apresentando grande potencial metastático para o desenvolvimento de metástases e altas taxas de morbimortalidade (Horto et al., 2020).

Em relação às terapias, pouco se mudou na abordagem terapêutica do CCP, sendo as mais utilizadas a ressecção cirúrgica, a radioterapia e a quimioterapia (com cisplatina, carboplatina, docetaxel, entre outros). Essa última, continua como uma alternativa bastante onerosa e provocando inúmeros efeitos colaterais nos pacientes (Rivera, 2015).

Neste contexto, o uso de compostos fenólicos pode representar uma possibilidade terapêutica com o objetivo de potencializar o tratamento do câncer. Polifenóis são metabólitos secundários encontrados em alimentos e em plantas medicinais. Cerca de mais de 8.000 compostos fenólicos naturais foram identificados até agora e são divididos em várias subclasses, como flavonóides, cumarinas, estilbenóides, lignanos, taninos e ácidos fenólicos (do Carmo et al., 2018). São candidatos cada vez mais comuns e requisitados para a obtenção de extratos e de drogas farmacologicamente ativas (House et al., 2020) com efeitos na promoção de saúde, da prevenção e do tratamento de doenças, incluindo o câncer (Arora et al., 2019).

Com resultados promissores em ensaios in vitro, in vivo e in silico, esses compostos se destacam como terapias alternativas complementares, adjuvantes e até mesmo suplementares no tratamento de diferentes condições, com atividade antiinflamatória, imunomoduladora e antineoplásica (Paraiso et al., 2020; Rayan et al., 2017). Além disso, os polifenóis (PFs) podem ser eficazes por produzir peróxido de hidrogênio e, com isso, induzir apoptose em células cancerosas por diferentes mecanismos, devido ao seu efeito pró-oxidante (do Carmo et al., 2018). Contudo, algumas limitações também são observadas, como atingir o aproveitamento adequado desses compostos e manutenção de sua viabilidade após processamento industrial dos alimentos (Olszewska et al., 2020), sua biodisponibilidade baixa (Arora et al., 2019) e o incompleto entendimento dos mecanismos farmacodinâmicos e farmacotécnicos que possibilitem comercialização desses como fármaco (Rein et al., 2013; Yan et al., 2020).

Portanto, considerando as diversas atividades biológicas apresentadas pelos polifenóis e a necessidade de buscar terapias alternativas e complementares para o tratamento do CCP, este estudo teve como objetivo identificar os efeitos do uso de polifenóis na terapia do câncer de cabeça e pescoço. 


\section{Metodologia}

Trata-se de revisão integrativa elaborada de acordo referencial metodológico (Souza et al., 2010) que abordam seis fases de pesquisa: 1) elaboração da pergunta norteadora; 2) busca nas bases de dados; 3) Coleta de dados; 4) Análise crítica dos estudos incluídos; 5) Discussão dos resultados e, 6) Apresentação dos dados, reportando de forma clara os resultados encontrados.

A pergunta norteadora deste estudo se baseou na estratégia P.I.Co (Kellen et al., 2019), em que o "P" corresponde ao problema "câncer de cabeça e pescoço", o "I" ao interesse "efeitos do uso de polifenóis" e "Co" ao contexto do estudo "terapia destes cânceres". Com isso, formulou-se a questão: "Quais os efeitos do uso de polifenóis na terapia do câncer de cabeça e pescoço?".

A fase de busca na literatura abordou as seguintes bases de dados: Medical Literature Analysis and Retrieval Sistem on-line (MEDLINE) a partir do motor de busca PubMed®; ScienceDirect; Scopus (SciVerse Scopus) e também foi consultada a Biblioteca Virtual de Saúde Brasil (BVS).

Para a seleção de artigos foram adotados os seguintes critérios de inclusão: Todas as categorias de artigos; com publicação nos idiomas português, inglês ou espanhol, publicados nos últimos 5 anos, visto que nesse período houve aumento expressivo no número de pesquisas envolvendo compostos naturais e câncer, e também que respondessem à questão norteadora do estudo.

Para a busca dos artigos foram utilizados os seguintes descritores reconhecidos pelo Descritores em Ciências da Saúde (DeCS) e pelo Medical Subject Headings (MeSH): "Polyphenols", "Head and Neck Cancer" e "Therapy". Foi usado o operador booleano "AND" para associar os descritores nas bases de dados e na BVS, sendo realizada nessa biblioteca a busca também pelos termos "Polifenois", "Câncer de Cabeça e Pescoço" e "Terapia"; bem como "Polifenoles", "Neoplasias de Cabeza e Cuello" e "Terapia".

Como estratégia de extração de dados, buscou-se informações relevantes no contexto de terapia: Identificação do composto fenólico utilizado e possíveis associações entre dois ou mais desses; o delineamento do estudo, para que fosse possível estabelecer o nível de evidência apresentado; e quais os principais resultados apresentados pelos autores, com destaque para os mecanismos celulares descritos.

A construção do banco de dados foi feita aplicando-se um "Instrumento de coleta de dados da revisão integrativa", com algumas adaptações, proposta por autores (Fernandes, 2011), contendo variáveis de identificação do estudo, assim como os principais resultados.

Para classificação do nível de evidência dos estudos, foi utilizado o referencial para hierarquização dos níveis de evidência proposto por STETLER et al. (1998) descritos a seguir:

-Nível 1: Evidências resultantes da meta-análise de múltiplos estudos clínicos controlados e randomizados;

-Nível 2: Evidências obtidas em estudos individuais com delineamento experimental;

- Nível 3: Evidências de estudos quase-experimentais;

-Nível 4: Evidências de estudos descritivos (não-experimentais) ou com abordagem qualitativa;

-Nível 5: Evidências provenientes de relatos de caso ou de experiência;

- Nível 6: Evidências baseadas em opiniões de especialistas.

\section{Resultados e Discussão}

\section{Resultados}

A Figura 1 apresenta as fases de identificação e seleção dos estudos encontrados na estratégia de busca. Ao final, 25 artigos que respondiam à pergunta norteadora, compuseram a amostra para síntese dessa revisão. 
Figura 1. Fluxograma PRISMA de identificação e de seleção dos estudos encontrados pela estratégia de busca.

\section{Identificação e análise dos estudos}

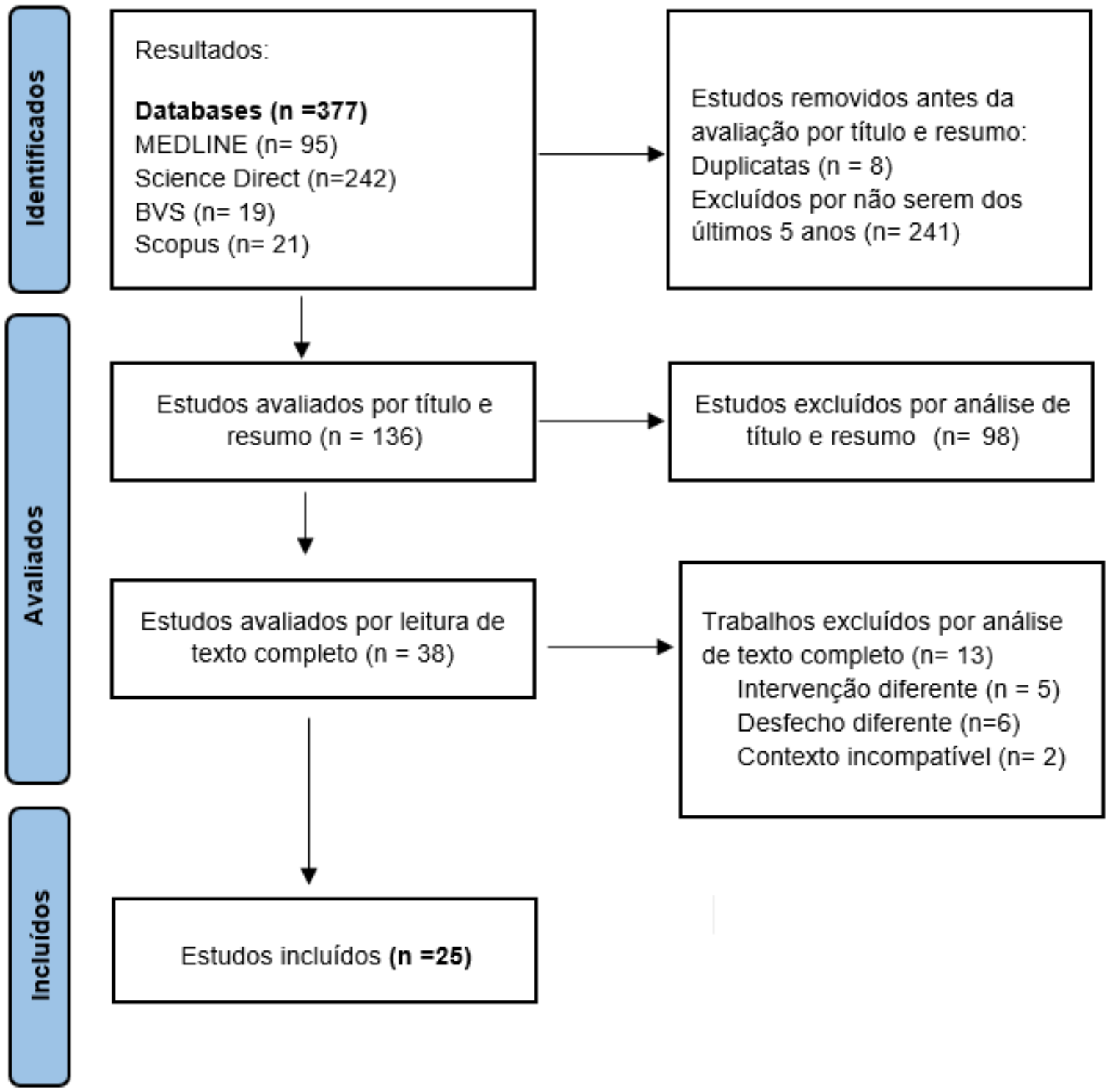

Fonte: Autores.

A análise dos estudos mostrou que o maior número de publicações está concentrado no ano de 2019 (n=7, 28\%). Em relação ao idioma, todos os artigos estavam na língua inglesa $(n=25,100 \%)$. Sobre o tipo de estudo realizado, a maioria dos artigos foram conduzidos no delineamento de revisões $(\mathrm{n}=10,40 \%)$ e estudos in vitro $(\mathrm{n}=10,40 \%)$.

Em relação aos níveis de evidência, 52\% dos artigos $(\mathrm{n}=13)$ apresentaram nível de evidência 2, 44\% (n=11) apresentaram nível 4 e apenas um artigo apresentou nível 5.

Dos compostos fenólicos apresentados nos estudos incluídos desta revisão, os que mais apareceram foram a Curcumina $(n=10,40 \%)$, Resveratrol $(n=10,40 \%)$ e Epicatequina-3-galato $(n=9,36 \%)$, sendo que alguns estudos incluíram a análise de dois ou mais compostos.

O Quadro 1 apresenta a síntese da estratégia de extração de dados dos artigos incluídos na revisão, descrevendo os compostos fenólicos e os principais resultados dos estudos referente ao tratamento de câncer de cabeça e de pescoço. 
Quadro 1. Síntese da estratégia de extração de dados dos artigos incluídos na revisão.

\begin{tabular}{|c|c|c|c|c|c|}
\hline ID & $\begin{array}{l}\text { Autores e ano de } \\
\text { publicação }\end{array}$ & Tipo de estudo & $\begin{array}{l}\text { Nível de } \\
\text { Evidência }\end{array}$ & Composto Fenólico & Principais resultados \\
\hline 1 & $\begin{array}{l}\text { Benvenuto M. et } \\
\text { al. - } 2016\end{array}$ & $\begin{array}{l}\text { Experimental (In } \\
\text { vivo) }\end{array}$ & 2 & Gossipol & $\begin{array}{l}\text { Inibição de proliferação celular, } \\
\text { indução de autofagia e apoptose. }\end{array}$ \\
\hline 2 & $\begin{array}{l}\text { Demiray, M.; et } \\
\text { al. } 2016\end{array}$ & Relato de Caso & 5 & Curcumina & $\begin{array}{c}\text { Inibição da proliferação celular e } \\
\text { diminuição da ativação constitutiva } \\
\text { das vias de sinalização de crescimento } \\
\text { de células cancerosas. }\end{array}$ \\
\hline 3 & $\begin{array}{l}\text { Inaba, H.; et al. } \\
2016\end{array}$ & $\begin{array}{l}\text { Experimental (In } \\
\text { vitro) }\end{array}$ & 2 & $\begin{array}{l}\text { Diversos tipos de } \\
\text { polifenóis }\end{array}$ & $\begin{array}{l}\text { Inibição da ativação de proMMP9 e } \\
\text { da invasão celular. }\end{array}$ \\
\hline 4 & $\begin{array}{l}\text { Katiyar S. K. - } \\
\quad 2016\end{array}$ & Revisão & 4 & $\begin{array}{l}\text { Proantocianidina, } \\
\text { Honokiol e } \\
\text { Epigalocatequina-3- } \\
\text { galato (EGCG) }\end{array}$ & $\begin{array}{c}\text { Redução de viabilidade celular e } \\
\text { indução de apoptose. Esses efeitos } \\
\text { foram associados a efeitos inibitórios } \\
\text { dos fitoquímicos sobre o receptor do } \\
\text { fator de crescimento epidérmico } \\
\text { (EGFR) e proteínas reguladoras do } \\
\text { ciclo celular. }\end{array}$ \\
\hline 5 & $\begin{array}{l}\text { Mohan A., et al. } \\
2016\end{array}$ & $\begin{array}{l}\text { Experimental (In } \\
\text { vitro) }\end{array}$ & 2 & Trans-Resveratrol & $\begin{array}{l}\text { Maior expressão de proteínas } \\
\text { relacionadas com ciclo celular e } \\
\text { indução de apoptose. }\end{array}$ \\
\hline 6 & $\begin{array}{l}\text { Niedzwiecki A. et } \\
\text { al. - } 2016\end{array}$ & Revisão & 4 & $\begin{array}{l}\text { PB, uma mistura de } \\
\text { quercetina, curcumina, } \\
\text { GTP (Polifenois do chá } \\
\text { verde), cruciferex e } \\
\text { resveratrol; curcumina, } \\
\text { resveratrol e } \\
\text { epicatequina galato }\end{array}$ & $\begin{array}{c}\text { Inibição de proliferação celular, } \\
\text { diminuição da viabilidade celular, } \\
\text { sobrevivência clorogênica e aumento } \\
\text { da apoptose. }\end{array}$ \\
\hline 7 & $\begin{array}{l}\text { Rauf A., et al., } \\
2016 \\
\end{array}$ & Revisão & 4 & Resveratrol & $\begin{array}{c}\text { Supressão de viabilidade celular e } \\
\text { promoção de apoptose. }\end{array}$ \\
\hline 8 & $\begin{array}{c}\text { Costa, C.; et al. } \\
2017\end{array}$ & Revisão & 4 & $\begin{array}{c}\text { Curcumina, } \\
\text { Epigalocatequina-3- } \\
\text { Galato (ECCG) e } \\
\text { Resveratrol }\end{array}$ & $\begin{array}{c}\text { Inibição da proliferação celular, } \\
\text { indução de apoptose e interrupção no } \\
\text { ciclo celular. }\end{array}$ \\
\hline 9 & $\begin{array}{l}\text { Kim, S.; et al, } \\
2017 .\end{array}$ & $\begin{array}{l}\text { Experimental (In } \\
\text { vitro) }\end{array}$ & 2 & Resveratrol & $\begin{array}{c}\text { Diminuição da viabilidade celular e } \\
\text { aumento da proporção de células } \\
\text { apoptóticas. }\end{array}$ \\
\hline 10 & $\begin{array}{l}\text { Kunnumakkara } \\
\text { A. B. et al. - } 2017\end{array}$ & Revisão & 4 & Curcumina & $\begin{array}{l}\text { Regulação do crescimento de células } \\
\text { tumorais e potencialização de agentes } \\
\text { anticâncer. }\end{array}$ \\
\hline 11 & $\begin{array}{l}\text { Pandey, M.; et al. } \\
2017\end{array}$ & Revisão & 4 & Curcumina & $\begin{array}{l}\text { Atividade anticâncer por meio da } \\
\text { inibição de EGFR (receptor do fator } \\
\text { de crescimento epidérmico), }\end{array}$ \\
\hline 12 & $\begin{array}{l}\text { Shakya G., et al., } \\
2017\end{array}$ & $\begin{array}{l}\text { Experimental (In } \\
\text { vitro e in silico) }\end{array}$ & 2 & $\begin{array}{l}\text { MEWG (rutina, } \\
\text { quercetina, ácido } \\
\text { gálico, ácido ferúlico, e } \\
\text { ácido cafeico) }\end{array}$ & Inibição de metástase e angiogênese. \\
\hline 13 & $\begin{array}{l}\text { Srinivasan, M.;et } \\
\text { al. } 2017\end{array}$ & Revisão & 4 & Curcumina & $\begin{array}{l}\text { Inibição a proliferação, migração, } \\
\text { invasão e metástase e induz apoptose. }\end{array}$ \\
\hline 14 & $\begin{array}{l}\text { Tan y., et al., } \\
2017\end{array}$ & $\begin{array}{l}\text { Experimental (In } \\
\text { vivo e in vitro) }\end{array}$ & 2 & Resveratrol & $\begin{array}{c}\text { Parada do ciclo celular, diminuiçãa de } \\
\text { volume e peso tumoral. Demostrou } \\
\text { efeito radiosensibilizador do } \\
\text { composto. }\end{array}$ \\
\hline 15 & $\begin{array}{c}\text { Chakraborty, P.; } \\
\text { et al. } 2018 \\
\end{array}$ & Revisão & 4 & $\begin{array}{c}\text { Epigalocatequina-3- } \\
\text { Galato (ECCG) }\end{array}$ & $\begin{array}{l}\text { Inibição da invasão e motilidade das } \\
\text { células. }\end{array}$ \\
\hline 16 & $\begin{array}{l}\text { Mukherjee, S.; et } \\
\text { al. } 2018\end{array}$ & $\begin{array}{l}\text { Experimental } \\
\quad \text { (In vitro) }\end{array}$ & 2 & $\begin{array}{l}\text { TriCurin (Formulação } \\
\text { de curcumina, } \\
\text { resveratrol e } \\
\text { epicatequinagalato) }\end{array}$ & $\begin{array}{l}\text { Estimula o sistema imunológico } \\
\text { contra células cancerosas e }\end{array}$ \\
\hline
\end{tabular}




\begin{tabular}{|c|c|c|c|c|c|}
\hline 17 & $\begin{array}{l}\text { Sheng H., et al., } \\
2018\end{array}$ & $\begin{array}{l}\text { Experimental } \\
\text { (In vitro) }\end{array}$ & 2 & $\begin{array}{c}\text { Resveratrol, } \\
\text { epigalocatequina } \\
\text { galato, ácido tanico }\end{array}$ & $\begin{array}{c}\text { Aumento de citotoxicidade e } \\
\text { seletividade, promovendo apoptose }\end{array}$ \\
\hline 18 & $\begin{array}{l}\text { Belobrov S. et al. } \\
\quad-2019\end{array}$ & $\begin{array}{l}\text { Experimental } \\
\quad \text { (In vitro) }\end{array}$ & 2 & EGCG & $\begin{array}{l}\text { Redução de proliferação celular e de } \\
\text { migração celular, e diminuição de } \\
\text { expressão da forma fosforilada de } \\
\text { EGFR. }\end{array}$ \\
\hline 19 & $\begin{array}{l}\text { Bisol A., de } \\
\text { Campos P.S. e } \\
\text { Lamers M. L. - } \\
2019 \\
\end{array}$ & Revisão & 4 & $\begin{array}{l}\text { Ácido acético de } \\
\text { flavona }\end{array}$ & $\begin{array}{l}\text { Estabilização da doença, sem } \\
\text { resultados clinicamente relevantes. }\end{array}$ \\
\hline 20 & $\begin{array}{l}\text { Lai, K.; et al. } \\
\quad 2019\end{array}$ & $\begin{array}{l}\text { Experimental } \\
\text { (In vivo e in } \\
\text { vitro })\end{array}$ & 2 & Curcumina & Indução de apoptose. \\
\hline 21 & $\begin{array}{l}\text { Liang, Y.; et al. } \\
2019\end{array}$ & $\begin{array}{l}\text { Experimental } \\
\quad \text { (In vitro) }\end{array}$ & 2 & $\begin{array}{l}\text { O éster fenetílico do } \\
\text { ácido cafeico (CAPE) }\end{array}$ & $\begin{array}{c}\text { Supressão a viabilidade celular e } \\
\text { provocou apoptose. Tratamento CAPE } \\
\text { agiu sinergicamente com } \\
\text { quimioterapia e radioterapia. }\end{array}$ \\
\hline 22 & $\begin{array}{l}\text { López, E. L.; } \\
\text { Garcia F. G.; } \\
\text { Jornet, P. L.; } \\
\quad 2019 \\
\end{array}$ & $\begin{array}{l}\text { Experimental } \\
\quad \text { (In vitro) }\end{array}$ & 2 & $\begin{array}{l}\text { Epigalocatequina-3- } \\
\text { Galato (ECCG) }\end{array}$ & $\begin{array}{l}\text { Redução da viabilidade celular em } \\
\text { combinação com o quimioterápico. }\end{array}$ \\
\hline 23 & $\begin{array}{l}\text { Mikami, S.; et al. } \\
2019\end{array}$ & $\begin{array}{l}\text { Experimental } \\
\quad \text { (In vivo) }\end{array}$ & 2 & $\begin{array}{l}\text { Resveratrol }(3,4 \text { ', } \\
5 \text { - trihidroxistilbeno) }\end{array}$ & $\begin{array}{l}\text { Aumentou o efeito antitumoral da } \\
\text { radiação ou cisplatina } \\
\text { in vivo. }\end{array}$ \\
\hline 24 & $\begin{array}{l}\text { Rafiee, Z. et al. } \\
2019\end{array}$ & Revisão & 4 & Curcumina & Inibição da proliferação celular. \\
\hline 25 & $\begin{array}{l}\text { Khatoon, E.; et al. } \\
2020\end{array}$ & Revisão & 4 & $\begin{array}{l}\text { Apigenina, Curcumina, } \\
\text { Epigalocatequina-3- } \\
\text { Galato (EGCG) e } \\
\text { Quercetina } \\
\end{array}$ & Inibição da proliferação celular. \\
\hline
\end{tabular}

Fonte: Autores.

\section{Discussão}

O Câncer de Cabeça e Pescoço ainda é uma condição que apresenta altas taxas de morbimortalidade, mesmo com os avanços das terapias convencionais (Kim et al., 2017). Esse tipo de câncer é caracterizado pela invasividade, grandes chances de recidiva precoce e frequentemente pela presença de metástase em linfonodos. Além disso, essas condições também são consequências clínicas diretamente relacionadas com biomarcadores que modulam a malignidade (Inaba et al., 2016; Kim et al., 2017). Desde a carcinogênese, em que ocorre perturbação de diversas vias de sinalizações celulares (Kunnumakkara et al., 2017), até a metástase, que manifesta algum grau de resistência a terapias convencionais (Shakya et al., 2018), há envolvimento ativo de mutações genéticas e somáticas ou então mudanças epigenéticas relacionadas ao estilo de vida e alterações ambientais (Pandey et al., 2017). Esse conjunto de distúrbios celulares e moleculares pode refletir diretamente na manifestação clínica do CCP, com grande parte dos prognósticos sendo desfavoráveis, apresentando alta complexidade de tratamento (Inaba et al., 2016).

$\mathrm{Na}$ abordagem dos métodos de terapia dos $\mathrm{CCP}$, a literatura tem intensificado suas análises para componentes bioativos do reino vegetal, que podem ser capazes de oferecer novas opções para o desenvolvimento de quimioterápicos ou coadjuvantes para terapias citotóxicas convencionais (Katiyar, 2016). Os polifenóis apresentaram boa resposta nesse quesito, sendo capazes de atuarem em sítios e momentos específicos da carcinogênese, apresentando respostas na modulação das vias de sinalização celular, transcrição de DNA, expressão gênica, proliferação e sobrevivência celular (Niedzwiecki et al., 2016).

Nesse sentido, os achados com a utilização de polifenóis apontam para uma redução da viabilidade celular de diversas linhagens de CCP. Mukherjee (2018) encontrou esse efeito com o uso de uma combinação de curcumina, resveratrol e EGGC (TriCurin) que atua de forma eficaz na estimulação do sistema imunológico contra metabolismos de células cancerosas. Além 
disso, essa composição apresenta redução de viabilidade seletiva para linhagens cancerosas não afetando células normais. Sheng et al. (2018) também mostrou que o uso do revesratrol, da EGGC e do ácido tânico aumentou a citotoxidade da Doxorrubicina, um medicamento antineoplásico. Esses resultados, principalmente relacionados com o uso de EGGC corroboram com os estudos de Belobrov (2019); Lopez (2019) e Khatoon (2020). Foram encontrados efeitos dose dependentes e o mecanismo pode estar relacionado com o aumento da apoptose gerado pela elevação de Espécies Reativas de Oxigênio (ERO) induzidas pela oxidação dos polifenóis, podendo ser classificados, neste contexto, como compostos pró-oxidantes (Sheng et al., 2018).

Um estudo demonstrou a supressão da viabilidade celular associado ao isso de polifenóis. Entre eles pode-se destacar que uma porção significativa dos cânceres de cabeça e pescoço expressam altos índices de BCl-2 anti-apoptótica, que está diretamente relacionada com resistência à quimioterapia e à radioterapia, resultado em baixa eficácia dos tratamentos clínicos para estas condições (Benvenuto et al., 2017). Diretamente relacionada com a BCl-2, a Bax favorece a via oposta, sendo pró apoptótica e reguladora da via intrínseca de apoptose. A taxa $\mathrm{Bax} / \mathrm{BCl} 2$ é essencial para a modulação do favorecimento ou não da apoptose nas células tumorais (Kim et al., 2017). Quanto a isso, diferentes polifenóis apresentaram resultados importantes quanto à redução de $\mathrm{BCl} 2$ e aumento de Bax, favorecendo a via de morte celular como o gossipol, polifenol alcaloide encontrado na semente de algodão (Benvenuto et al., 2017); resveratrol (Kim et al., 2017); Honokiol, um polifenol lignano abundante em plantas do gênero Magnolia; ácido cafeico (Liang et al., 2019), curcumina, EGGC e revestratrol (Costa et al., 2017). Relacionado aos últimos compostos citados, Rauf et al. (2016) percebeu em seu estudo efeitos na indução de apoptose devido a alteração na taxa Bax/Bcl2 com a associação entre curcumina e resveratrol, indicando um efeito sinérgico entre as duas substâncias, o que pode potencializar o protocolo de tratamento.

Outro fator importante é a ação de modulação negativa desses compostos na via envolvendo NF-kB, o que desfavorece a manutenção do microambiente tumoral e pró-inflamatório (Katiyar, 2016). Esse complexo proteico possui função na a iniciação do câncer humano, desenvolvimento, supressão de apoptose, e favorecimento de angiogênese e metástase, que clinicamente pode se caracterizar como resistência ao tratamento. Deste modo, compostos que suprimam a ação do NF-אB podem trazer avanços na pesquisa relacionada ao CCP, como no estudo de Katiyar (2016) que demonstrou que o gossipol é capaz de inativar essa via. Resultados de inibição desse mecanismo também foram encontrados utilizando curcumina (Khatoon et al., 2020; Kunnumakkara et al., 2017; Rafiee et al., 2019; Srinivasan, 2017), inclusive com associação entre a curcumina e a cisplatina, um medicamento antineoplásico, que resultou em capacidade de diminuição da quimiorresistência das células e potencialização do efeito do quimioterápico. Outros autores também observaram efeitos no controle do estresse oxidativo pela modulação de NF-kB, quando utilizando o ácido cafeico (Liang et al., 2019) e uma combinação de curcumina e resveratrol (Rauf et al., 2016).

Essa combinação de resveratrol e curcumina também foi responsável pela inibição de expressão de metaloproteinases (MMP), principalmente a 9, sob diferentes doses (Rauf et al., 2016). O desenvolvimento e gravidade do CCP estão diretamente associados com a expressão de MMPs, especialmente a 2, 7 e 9, que em associação com patógenos periodontais, como a $P$. gingivalis (Inaba et al., 2016) denota maior taxa de metástase e mortalidade. Também foi relatado a diminuição de MMP de forma dose-dependente, por intermediação das proteínas pró-apoptóticas Bax, com uso de diferentes polifenóis (Inaba et al., 2016; Khatoon et al., 2020; Kim et al., 2017; Niedzwiecki et al., 2016; Shakya et al., 2018).

Além das vias citadas, o potencial de migração e de invasividade das células tumorais, determinado pelo processo de Transição Epitélio-Mesenquimal (TEM), está diretamente relacionado com a capacidade de metástase dos CCP e atua como fator de malignidade. Neste sentido, Katiyar (2016) relatou a ação de proantocianidinas de sementes de uva (GSP) na desregulação de biomarcadores mesenquimais com consequente inativação, bem como com a reativação dos epiteliais, revertendo TEM, e inibindo migração de CCP. Esse resultado foi similar com o obtido com o uso de resveratrol (Kim et al., 2017) e de ácido cafeico (Liang et al., 2019). 
Apesar dos efeitos dos polifenóis nas vias de desenvolvimento e progressão do tumor, alguns pontos são importantes de serem compreendidos sobre desafios terapêuticos da utilização destes compostos. Primeiramente, estudos mostraram efeitos anticarcinogênicos ou pró-apoptóticos em condições dose dependente (Benvenuto et al., 2017; Inaba et al., 2016; Katiyar, 2016; Kim et al., 2017; Niedzwiecki et al., 2016; Tan et al., 2017), fato que diminui o potencial farmacológico, uma vez que grandes doses são necessárias e é esperado que crie-se uma relação frequente de aumento dessas para manutenção do efeito terapêutico. Além disso, é precisa destacar a baixa biodisponibilidade e/ou baixa solubilidade desses compostos (Kunnumakkara et al., 2017; Mohan et al., 2016). Para melhoria dessas características físico-químicas, sugere-se a associação de compostos polifenólicos com tecnologia que facilite a passagem transmembrana (Kunnumakkara et al., 2017) ou encapsulamentos (Demiray et al., 2016) para manter os efeitos terapêuticos desejados e potencializar biodisponibilidade (Srinivasan, 2017).

Vale ressaltar como limitações da presente revisão que a maioria dos estudos caracterizam nível de evidência 2, sendo desenvolvidos em modelos animais ou com culturas de células, que representam importante fonte de conhecimento molecular, mas que ainda refletem distanciamento do uso terapêutico efetivo em seres humanos.

Portanto, este estudo foi capaz de identificar os principais compostos fenólicos e seus potenciais efeitos antineoplásicos contra os CCP, atuando na cascata apoptótica e na redução da taxa de metástase. Estudos futuros devem caminhar no sentido de caracterizar os efeitos demostrados pelos compostos fenólicos, abordando suas dosagens e possíveis efeitos adversos para ampliação da discussão acerca deste assunto e contribuir para avanços relacionados a terapêutica dos CCP.

\section{Considerações Finais}

Sendo assim, abordando a malignidade dos cânceres de cabeça e pescoço e a necessidade de encontrar novas terapias que possam potencializar os efeitos da terapia convencional, os polifenóis curcumina, ECCG, resveratrol e ácido cafeico foram identificados como os compostos mais estudados nos trabalhos incluídos da presente revisão.

Entre mecanismos relacionados com a diminuição da proliferação celular, pode-se citar modulação da via de NF-kB, redução de BCl2 (antiapoptótica) e aumento de Bax (pró-apoptótica), inibição de expressão de metaloproteinases (MMP) e inibição do processo de Transição Epitélio-Mesenquimal, que contribui para migração dos CCP.

Tendo em vista que a maioria dos estudos trabalharam com modelos animais, cultura de células ou mesmo revisões de estudos laboratoriais, análises mais abrangentes devem ser incentivadas no sentido de detalhar as vias de ação e avaliar as condições de aplicação dos compostos fenólicos, bem como as possíveis doses e os efeitos adversos apresentados por esses compostos.

\section{Referências}

Alterio, D., Marvaso, G., Ferrari, A., Volpe, S., Orecchia, R., \& Jereczek-Fossa, B. A. (2019). Modern radiotherapy for head and neck cancer. Seminars in Oncology, 46(3), 233-245. https://doi.org/10.1053/j.seminoncol.2019.07.002

Arora, I., Sharma, M., \& Tollefsbol, T. O. (2019). Combinatorial epigenetics impact of polyphenols and phytochemicals in cancer prevention and therapy. International Journal of Molecular Sciences, 20(18). https://doi.org/10.3390/ijms20184567

Belobrov, S., Seers, C., Reynolds, E., Cirillo, N., \& McCullough, M. (2019). Functional and molecular effects of a green tea constituent on oral cancer cells. Journal of Oral Pathology \& Medicine : Official Publication of the International Association of Oral Pathologists and the American Academy of Oral Pathology, 48(7), 604-610. https://doi.org/10.1111/jop.12914

Benvenuto, M., Mattera, R., Masuelli, L., Taffera, G., Andracchio, O., Tresoldi, I., Lido, P., Giganti, M. G., Godos, J., Modesti, A., \& Bei, R. (2017). ( \pm )Gossypol induces apoptosis and autophagy in head and neck carcinoma cell lines and inhibits the growth of transplanted salivary gland cancer cells in BALB/c mice. International Journal of Food Sciences and Nutrition, 68(3), 298-312. https://doi.org/10.1080/09637486.2016.1236077

Cohen, N., Fedewa, S., \& Chen, A. Y. (2018). Epidemiology and Demographics of the Head and Neck Cancer Population. Oral and Maxillofacial Surgery Clinics of North America, 30(4), 381-395. https://doi.org/10.1016/j.coms.2018.06.001

Costa, C., Tsatsakis, A., Mamoulakis, C., Teodoro, M., Briguglio, G., Caruso, E., Tsoukalas, D., Margina, D., Dardiotis, E., Kouretas, D., \& Fenga, C. (2017). Current evidence on the effect of dietary polyphenols intake on chronic diseases. Food and Chemical Toxicology, 110, 286-299. https://doi.org/10.1016/j.fct.2017.10.023 
Demiray, M., Sahinbas, H., Atahan, S., Demiray, H., Selcuk, D., Yildirim, I., \& Atayoglu, A. T. (2016). Successful treatment of c-kit-positive metastatic Adenoid Cystic Carcinoma (ACC) with a combination of curcumin plus imatinib: A case report. Complementary Therapies in Medicine, 27, 108-113. https://doi.org/10.1016/j.ctim.2016.06.009

do Carmo, M. A. V., Pressete, C. G., Marques, M. J., Granato, D., \& Azevedo, L. (2018). Polyphenols as potential antiproliferative agents: scientific trends. Current Opinion in Food Science, 24, 26-35. https://doi.org/10.1016/j.cofs.2018.10.013

Fernandes, D. de L. (2011). Representações sociais de adolescentes sobre o câncer de colo do útero. Universidade Federal de Pernambuco.

Horto, J. D. H., Knochelmann, H. M., Day, T. A., Paulos, C. M., \& Neskey, D. M. (2020). Immune Evasion by Head and Neck Cancer: Foundations for Combination Therapy. Trends Cancer, 176(1), 139-148. https://doi.org/10.1016/j.trecan.2019.02.007.Immune

House, N. C., Puthenparampil, D., Malayil, D., \& Narayanankutty, A. (2020). Variation in the polyphenol composition, antioxidant, and anticancer activity among different Amaranthus species. South African Journal of Botany, 135, 408-412. https://doi.org/10.1016/j.sajb.2020.09.026

Inaba, H., Tagashira, M., Kanda, T., Murakami, Y., Amano, A., \& Matsumoto-Nakano, M. (2016). Apple- and Hop-Polyphenols Inhibit Porphyromonas gingivalis-Mediated Precursor of Matrix Metalloproteinase-9 Activation and Invasion of Oral Squamous Cell Carcinoma Cells. Journal of Periodontology, 87(9), 1103-1111. https://doi.org/10.1902/jop.2016.160047

Katiyar, S. K. (2016). Emerging Phytochemicals for the Prevention and Treatment of Head and Neck Cancer. Molecules (Basel, Switzerland), 21(12). https://doi.org/10.3390/molecules21121610

Kellen, C., Sousa, L., Catarina, A. N. A., Silva, D. E. O., Laiane, A. N. A., Silva, D. A., Carvalho, A. S., Mangueira, C. C., Pereira, L. L., Abreu, D. E., Patrícia, M., Cordeiro, L., \& Muniz, M. K. (2019). AS EVIDÊNCIAS CIENTÍFICAS DA BRONQUIECTASIA : ETIOLOGIA , DIAGNÓSTICO E FORMAS DE THE SCIENTIFIC EVIDENCE OF BRONCHIECTASIA : ETIOLOGY, DIAGNOSIS AND FORMS OF TREATMENT. 26, 78-83.

Khatoon, E., Banik, K., Harsha, C., Sailo, B. L., Thakur, K. K., Khwairakpam, A. D., Vikkurthi, R., Devi, T. B., Gupta, S. C., \& Kunnumakkara, A. B. (2020). Phytochemicals in cancer cell chemosensitization: Current knowledge and future perspectives. Seminars in Cancer Biology, November 2019, 1-34. https://doi.org/10.1016/j.semcancer.2020.06.014

Kim, S. E., Shin, S. H., Lee, J. Y., Kim, C. H., Chung, I. K., Kang, H. M., Park, H. R., Park, B. S., \& Kim, I. R. (2017). Resveratrol induces mitochondrial apoptosis and inhibits epithelial-mesenchymal transition in oral squamous cell carcinoma cells. Nutrition and Cancer, 70(1), 125-135. https://doi.org/10.1080/01635581.2018.1397708

Kunnumakkara, A. B., Bordoloi, D., Harsha, C., Banik, K., Gupta, S. C., \& Aggarwal, B. B. (2017). Curcumin mediates anticancer effects by modulating multiple cell signaling pathways. Clinical Science, 131(15), 1781-1799. https://doi.org/10.1042/CS20160935

Liang, Y., Feng, G., Wu, L., Zhong, S., Gao, X., Tong, Y., Cui, W., Qin, Y., Xu, W., Xiao, X., Zhang, Z., Huang, G., \& Zhou, X. (2019). Caffeic acid phenethyl ester suppressed growth and metastasis of nasopharyngeal carcinoma cells by inactivating the NF-kB pathway. Drug Design, Development and Therapy, 13 , 1335-1345. https://doi.org/10.2147/DDDT.S199182

Mohan, A., Narayanan, S., Balasubramanian, G., Sethuraman, S., \& Krishnan, U. M. (2016). Dual drug loaded nanoliposomal chemotherapy: A promising strategy for treatment of head and neck squamous cell carcinoma. European Journal of Pharmaceutics and Biopharmaceutics: Official Journal of Arbeitsgemeinschaft Fur Pharmazeutische Verfahrenstechnik e.V, 99, 73-83. https://doi.org/10.1016/j.ejpb.2015.11.017

Mukherjee, S., Hussaini, R., White, R., Atwi, D., Fried, A., Sampat, S., Piao, L., Pan, Q., \& Banerjee, P. (2018). TriCurin, a synergistic formulation of curcumin, resveratrol, and epicatechin gallate, repolarizes tumor-associated macrophages and triggers an immune response to cause suppression of HPV+ tumors. Cancer Immunology, Immunotherapy, 67(5), 761-774. https://doi.org/10.1007/s00262-018-2130-3

Niedzwiecki, A., Roomi, M. W., Kalinovsky, T., \& Rath, M. (2016). Anticancer Efficacy of Polyphenols and Their Combinations. Nutrients, 8(9). https://doi.org/10.3390/nu8090552

Olszewska, M. A., Gędas, A., \& Simões, M. (2020). Antimicrobial polyphenol-rich extracts: Applications and limitations in the food industry. Food Research International, 134(April), 109214. https://doi.org/10.1016/j.foodres.2020.109214

Pandey, M. K., Gupta, S. C., Nabavizadeh, A., \& Aggarwal, B. B. (2017). Regulation of cell signaling pathways by dietary agents for cancer prevention and treatment. Seminars in Cancer Biology, 46(July), 158-181. https://doi.org/10.1016/j.semcancer.2017.07.002

Paraiso, I. L., Revel, J. S., \& Stevens, J. F. (2020). Potential use of polyphenols in the battle against COVID-19. Current Opinion in Food Science, 32, 149-155. https://doi.org/10.1016/j.cofs.2020.08.004

Pons-Fuster López, E., Gómez García, F., \& López Jornet, P. (2019). Combination of 5-Florouracil and polyphenol EGCG exerts suppressive effects on oral cancer cells exposed to radiation. Archives of Oral Biology, 101, 8-12. https://doi.org/10.1016/j.archoralbio.2019.02.018

Rafiee, Z., Nejatian, M., Daeihamed, M., \& Jafari, S. M. (2019). Application of curcumin-loaded nanocarriers for food, drug and cosmetic purposes. Trends in Food Science and Technology, 88(December 2018), 445-458. https://doi.org/10.1016/j.tifs.2019.04.017

Rauf, A., Imran, M., Sadiq Butt, M., Nadeem, M., Peters, D. G., \& Mubarak, M. S. (2016). Resveratrol as an Anticancer Agent: A Review A. Phytomedicine, 2016(5), 566-577.

Rayan, A., Raiyn, J., \& Falah, M. (2017). Nature is the best source of anticancer drugs: Indexing natural products for their anticancer bioactivity. PLoS ONE, 12(11), 1-12. https://doi.org/10.1371/journal.pone.0187925

Rein, M. J., Renouf, M., Cruz-Hernandez, C., Actis-Goretta, L., Thakkar, S. K., \& da Silva Pinto, M. (2013). Bioavailability of bioactive food compounds: A challenging journey to bioefficacy. British Journal of Clinical Pharmacology, 75(3), 588-602. https://doi.org/10.1111/j.1365-2125.2012.04425.x 
Research, Society and Development, v. 10, n. 9, e15210917804, 2021

(CC BY 4.0) | ISSN 2525-3409 | DOI: http://dx.doi.org/10.33448/rsd-v10i9.17804

Rivera, C. (2015). Essentials of oral cancer. International Journal of Clinical and Experimental Pathology, 8(9), 11884-11894. https://doi.org/10.5281/zenodo.192487

Shakya, G., Balasubramanian, S., Hoda, M., \& Rajagopalan, R. (2018). Inhibition of metastasis and angiogenesis in Hep-2 cells by wheatgrass extract - an in vitro and in silico approach. Toxicology Mechanisms and Methods, 28(3), 205-218. https://doi.org/10.1080/15376516.2017.1388460

Sheng, H., Ogawa, T., Niwano, Y., Sasaki, K., \& Tachibana, K. (2018). Effects of polyphenols on doxorubicin-induced oral keratinocyte cytotoxicity and anticancer potency against oral cancer cells. Journal of Oral Pathology \& Medicine : Official Publication of the International Association of Oral Pathologists and the American Academy of Oral Pathology, 47(4), 368-374. https://doi.org/10.1111/jop.12685

Souza, M. T. de, Silva, M. D. da, \& Carvalho, R. de. (2010). Revisão integrativa: o que é e como fazer. Reme: Revista Mineira de Enfermagem, 8(1), 102-106. https://doi.org/10.1590/S1679-45082010RW1134

Srinivasan, K. (2017). Antimutagenic and cancer preventive potential of culinary spices and their bioactive compounds. PharmaNutrition, 5(3), 89-102. https://doi.org/10.1016/j.phanu.2017.06.001

Stetler, C. B., Morsi, D., Rucki, S., Broughton, S., Corrigan, B., Fitzgerald, J., Giuliano, K., Havener, P., \& Sheridan, E. A. (1998). Utilization-Focused Integrative Reviews in a Nursing Service. 11(4), 195-206.

Tan, Y., Wei, X., Zhang, W., Wang, X., Wang, K., Du, B., \& Xiao, J. (2017). Resveratrol enhances the radiosensitivity of nasopharyngeal carcinoma cells by downregulating E2F1. Oncology Reports, 37(3), 1833-1841. https://doi.org/10.3892/or.2017.5413

Yan, Z., Zhong, Y., Duan, Y., Chen, Q., \& Li, F. (2020). Antioxidant mechanism of tea polyphenols and its impact on health benefits. Animal Nutrition, 6(2), 115-123. https://doi.org/10.1016/j.aninu.2020.01.001 